\title{
Padé approximants for the acoustical characteristics of rigid frame porous media
}

\author{
Article \\ Published Version
}

Chandler-Wilde, S. N. (1995) Padé approximants for the acoustical characteristics of rigid frame porous media. Journal of the Acoustical Society of America, 98 (2). pp. 1119-1129. ISSN 0001-4966 doi: https://doi.org/10.1121/1.413610 Available at https://centaur.reading.ac.uk/32665/

It is advisable to refer to the publisher's version if you intend to cite from the work. See Guidance on citing.

Published version at: http://dx.doi.org/10.1121/1.413610

To link to this article DOI: http://dx.doi.org/10.1121/1.413610

Publisher: Acoustical Society of America

All outputs in CentAUR are protected by Intellectual Property Rights law, including copyright law. Copyright and IPR is retained by the creators or other copyright holders. Terms and conditions for use of this material are defined in the End User Agreement.

\section{www.reading.ac.uk/centaur}

\section{CentAUR}

Central Archive at the University of Reading

Reading's research outputs online 


\title{
Padé approximants for the acoustical characteristics of rigid frame porous media
}

\author{
S. N. Chandler-Wilde \\ Department of Mathematics and Statistics, Brunel University, Uxbridge, Middlesex UB8 3PH, England \\ K. V. Horoshenkov \\ Department of Civil Engineering, University of Bradford, Bradford, West Yorkshire BD7 1DP, England
}

(Received 6 September 1994; revised 9 February 1995; accepted 13 March 1995)

\begin{abstract}
In this paper it is shown that a number of theoretical models of the acoustical properties of rigid frame porous media, especially those involving ratios of Bessel functions of complex argument, can be accurately approximated and greatly simplified by the use of Padé approximation techniques. In the case of the model of Attenborough [J. Acoust. Soc. Am. 81, 93-102 (1987)] rational approximations are produced for the characteristic impedance, propagation constant, dynamic compressibility, and dynamic density, as a function of frequency and the material parameters. The model proposed by Stinson and Champoux [J. Acoust. Soc. Am. 91, 685-695 (1992)] is approximated via rational approximation for the viscosity correction function. (C) 1995 Acoustical Society of America.
\end{abstract}

PACS numbers: 43.28.En, 43.20.Gp, 43.20.Jr, 43.55.Ev

$\begin{array}{ll}\text { LIST OF SYMBOLS } \\ C_{b} & \begin{array}{l}\text { complex compressibility of the bulk me- } \\ \text { dium }\end{array} \\ c & \text { adiabatic speed of sound } \\ d & \text { depth of porous layer } \\ d_{e}=q d & \text { effective depth of porous layer } \\ F(z)=(z / 4) I_{1}(z) /\left[I_{0}(z)-I_{1}(z)\right] & \text { modified viscosity correction function } \\ F^{+}, F^{-} & \text {viscosity correction functions for } e^{i \omega t} \\ F_{A} & e^{-i \omega t} \text { time dependences } \\ f & \text { rational approximation to } F \\ g_{C} & \text { frequency } \\ G_{C} & \text { compressibility function } \\ g_{\beta} & \text { rational approximation to the compressibil- } \\ G_{\beta} & \text { ity function } \\ & \text { admittance function } \\ g_{k} & \text { rational approximation to the admittance } \\ G_{k} & \text { function } \\ & \text { wave-number function } \\ g_{\rho} & \text { rational approximation to the wave-number } \\ G_{\rho} & \text { function } \\ I_{0}, I_{1} & \text { density function } \\ J_{0}, J_{1} & \text { rational approximation to the density func- } \\ & \text { tion }\end{array}$

\section{INTRODUCTION}

The variety of recent papers dedicated to the problem of sound propagation through porous materials displays the continued interest in the theoretical description of the acoustical properties of absorbent materials, particularly as the basis for noise control applications. ${ }^{1-7}$

A standard approach for propagation in a rigid framed porous material has been to model the fluid-filled medium as $k \quad$ wave number in fluid

$k_{b} \quad$ dynamic complex wave number of the bulk

$\begin{array}{ll}N_{\text {Pr }} & \text { medium } \\ P_{0} & \text { Prandtl number }\end{array}$

$P_{0} \quad$ static pressure

$q \quad$ tortuosity

$r=\Omega / q \quad$ reduced porosity

$S(z)=2 I_{1}(z) /\left[z I_{0}(z)\right]$

$s \quad$ Stinson and Champoux shape factor

$s_{p} \quad$ Attenborough pore shape factor

$t \quad$ time

$Z_{b} \quad$ dynamic complex impedance of the bulk medium

$\beta_{b}=\left(\rho_{0} c\right) / Z_{b} \quad$ normalized characteristic admittance of the bulk medium

$\beta_{s} \quad$ normalized surface admittance

$\gamma \quad$ ratio of specific heats

$\lambda_{A} \quad$ see Eqs. (12) and (41)

$\lambda_{s} \quad$ see Eq. (45)

$\rho_{b} \quad$ dynamic complex density of the bulk medium

$\begin{array}{ll}\rho_{0} & \text { density of fluid } \\ \sigma & \text { flow resistivity }\end{array}$

$\sigma_{e} \quad$ effective flow resistivity

$\Omega \quad$ porosity

$\omega \quad$ angular frequency 
ous pores of arbitrary shape, proposed theoretical formulas expressing $Z_{b}$ and $k_{b}$ (and also complex density $\rho_{b}$ and compressibility $C_{b}$ ) as a function of frequency and of the material's porosity, flow resistivity, tortuosity, and pore shape factor.

Recently, Stinson and Champoux ${ }^{4}$ have shown that the pore shape factor itself in the Attenborough formulas should be chosen to be frequency dependent. Stinson and Champoux proposed a modified version of the Attenborough model, incorporating the cross-sectional shape of the pores in the medium in a different way, with a shape factor which depends only on the pore shape and is frequency independent.

Both these models, of Attenborough and of Stinson and Champoux, while soundly based and showing excellent agreement with experimental measurement (see especially the detailed experiments in Ref. 4), are more complex and difficult to compute than the simple empirical model of Delany and $\mathrm{Bazley}^{8}$ (and see $\mathrm{Miki}^{2}$ ), or the more recent semiempirical models of Allard and Champoux ${ }^{5}$ and Wilson, ${ }^{6}$ in that they involve computing a ratio of Bessel functions, $J_{1}(z) / J_{0}(z)$, for complex values of $z$, with argument $\pm \pi / 4$. This extra complexity makes use of the models more difficult in terms of implementation, and computation with the models more time consuming. (The CPU time required to compute $Z_{b}$ and $k_{b}$ as functions of frequency and material parameters is an important consideration, for example, in inverse problems, where an attempt is made through some iterative numerical process to determine material parameters to fit observed acoustical data.) Furthermore, existing library routines for computation of $J_{0}$ and $J_{1}$ are usually limited in the argument range in which they can accurately estimate these functions due to the rapid growth and oscillations of $J_{0}(z)$ and $J_{1}(z)$ as $|z|$ increases with $\arg z$ $= \pm \pi / 4$.

In this paper we propose simple but accurate rational approximations for $Z_{b}, k_{b}, \rho_{b}$, and $C_{b}$ as a function of frequency in the case of the Attenborough model (Sec. II), and for the viscosity correction function in the case of the Stinson and Champoux model. The approximations proposed enable both models to be accurately and efficiently computed for all combinations of parameter values. The main feature of the approximations is that, in each case, they capture the first few terms of the asymptotic behavior for both low and high frequency exactly, and, at the same time, provide a good degree of approximation at intermediate-frequency ranges.

\section{THE ATTENBOROUGH MODEL}

We present below the expressions derived by Attenborough $^{9-11}$ for the frequency-dependent dynamic complex density $\left(\rho_{b}\right)$, compressibility $\left(C_{b}\right)$, impedance $\left(Z_{b}\right)$, and wave number $\left(k_{b}\right)$, for propagation of acoustic waves in a fluid-filled rigid porous medium. These equations are a development of an approximate analysis by Zwikker and Kosten, ${ }^{12}$ Zwikker and Kosten's analysis based on a model microstructure of parallel identical cylindrical pores of circular cross section with viscous and thermal flow effects treated separately. (The validity of Zwikker and Kosten's approach has recently been demonstrated by Stinson. ${ }^{3}$ ) We choose to express Attenborough's formulas in a new but equivalent form which makes change of time dependence from $e^{-i \omega t}$ to $e^{i \omega t}$ trivial, aids construction of approximations to Attenborough's model, and has other advantages mentioned below.

We introduce first some complex-valued functions of a complex variable $z$. Let

$$
S(z):=\frac{2 J_{1}(i z)}{i z J_{0}(i z)}=\frac{2 I_{1}(z)}{z I_{0}(z)},
$$

where, as usual, := denotes "is defined as" and $J_{0}$ and $J_{1}$ are the Bessel functions of zeroth and first order, respectively, and $I_{0}$ and $I_{1}$ are the corresponding modified Bessel functions (see Ref. 13). Let

$$
\begin{aligned}
& g_{\rho}(z):=[1-S(z)]^{-1}, \\
& g_{C}(z):=1+(\gamma-1) S\left(N_{\mathrm{Pr}_{\mathrm{r}}}^{1 / 2}\right),
\end{aligned}
$$

where $\gamma, N_{\mathrm{Pr}}$ were previously defined. Let

$$
g_{\beta}(z):=\sqrt{g_{C}(z) / g_{\rho}(z)}
$$

and

$$
g_{k}(z):=\sqrt{g_{C}(z) g_{\rho}(z)},
$$

where the square roots (and all other square roots of complex quantities in the paper) are taken with positive real part.

In terms of the above functions it is easy to see that Attenborough's equations can be written as

$$
\begin{aligned}
& \rho_{b} / \rho_{0}=\left(q^{2} / \Omega\right) g_{\rho}\left(\sqrt{-i} \lambda_{A}\right), \\
& C_{b}=\left(\Omega / \gamma P_{0}\right) g_{C}\left(\sqrt{-i} \lambda_{A}\right) .
\end{aligned}
$$

Further, since $\gamma P_{0}=\rho_{0} c^{2}$ and

$$
\begin{aligned}
& \omega \rho_{b}=k_{b} Z_{b}, \\
& \omega C_{b}=k_{b} / Z_{b},
\end{aligned}
$$

the normalized characteristic admittance of the porous medium, $\beta_{b}=\rho_{0} c / Z_{b}$, is given by

$$
\beta_{b}=(\Omega / q) g_{\beta}\left(\sqrt{-i} \lambda_{A}\right),
$$

and the wave number by

$$
k_{b} / k=q g_{k}\left(\sqrt{-i} \lambda_{A}\right) \text {. }
$$

In these equations $\Omega<1$ is the porosity of the porous material and $q>1$ the tortuosity. The dimensionless parameter $\lambda_{A}$ is given by

$$
\lambda_{A}=\left(1 / 2 s_{p}\right)\left(8 \rho_{0} q^{2} \omega / \Omega \sigma\right)^{1 / 2},
$$

where $s_{p}$ is the pore shape factor ratio, $\sigma$ the flow resistivity, and the other notations were previously defined.

Equations (6)-(11) are correct only for time dependence $e^{-i \omega t}$ but the change of time dependence to $e^{i \omega t}$ is straightforward. Since $(S(z))^{*}=S\left(z^{*}\right)$ (where $z^{*}$ denotes the complex conjugate of $z$ ) and the same relation holds for the functions $g_{\rho}, g_{C}, g_{\beta}$, and $g_{k}$, to convert the above equations (6), (7), (10), and (11) to $e^{i \omega t}$ time dependence it is necessary merely to replace $-i$ by $i$ throughout.

Note that, if the tortuosity and porosity take their limiting values $q=\Omega=1$, then the functions $g_{\rho}\left(\sqrt{-i} \lambda_{A}\right)$, 
$g_{C}\left(\sqrt{-i} \lambda_{A}\right), g_{\beta}\left(\sqrt{-i} \lambda_{A}\right)$, and $g_{k}\left(\sqrt{-i} \lambda_{A}\right)$ are the normalized density, compressibility, admittance, and wave number of the porous medium, respectively. Moreover, for arbitrary $q>1$ and $\Omega<1$ these functions are proportional to their respective acoustic variables for a given porous material. For this reason we will refer to $g_{\rho}, g_{C}, g_{\beta}$, and $g_{k}$ as the density, compressibility, admittance, and wave-number functions, respectively.

Note also that the above way of writing the Attenborough model makes it clear that, although four physical variables appear in the model $\left(q, \Omega, s_{p}, \sigma\right)$, the acoustic properties of the medium $\left(\rho_{b}, C_{b}, Z_{b}, k_{b}\right)$ depend on these variables only in the dimensionless combinations $q, \Omega$, and $\lambda_{A}$, as pointed out by Sabatier et al. ${ }^{14}$ Thus only $q, \Omega$, and $\lambda_{A}$ can be determined from acoustic measurements alone, and if only acoustic measurement of $Z_{b}$ is carried out, then only $\Omega / q$ and $\lambda_{A}$ can be determined.

\section{A. Low- and high-frequency approximations}

To obtain simpler approximate formulas for low and high frequencies we consider the behavior of $S(z)$ for a small and large argument, respectively. From Ref. 13, expression (9.6.10) it follows that, as $z \rightarrow 0$,

$$
S(z)=1-z^{2} / 8+z^{4} / 48+O\left(z^{6}\right),
$$

while, from Ref. 13, expression (9.7.1), as $z \rightarrow \infty$,

$$
S(z)=2 / z-1 / z^{2}+O\left(z^{-3}\right), \quad|\arg z|<\pi / 2 .
$$

From these formulas and Eqs. (2)-(5) it follows that

$g_{\beta}(z)=\left\{\begin{array}{l}w_{1}\left(z-w_{2} z^{3}\right)+O\left(z^{5}\right), \quad z \rightarrow 0, \\ 1-w_{3} z^{-1}+O\left(z^{-2}\right), \quad z \rightarrow \infty, \quad|\arg z|<\pi / 2,\end{array}\right.$

where

$w_{1}:=\sqrt{\frac{\gamma}{8}}, w_{2}:=\frac{1}{2}\left(\frac{(\gamma-1) N_{\mathrm{Pr}}}{8 \gamma}+\frac{1}{6}\right), w_{3}:=1-\frac{\gamma-1}{N_{\mathrm{Pr}}}$.

Also

$g_{k}(z)=\left\{\begin{array}{l}\tilde{w}_{1}\left(z^{-1}+\tilde{w}_{2} z\right)+O\left(z^{3}\right), \quad z \rightarrow 0, \\ 1+\tilde{w}_{3} z^{-1}+O\left(z^{-2}\right), \quad z \rightarrow \infty, \quad|\arg z|<\pi / 2,\end{array}\right.$

where

$$
\begin{aligned}
& \tilde{w}_{1}:=\sqrt{8 \gamma}, \quad \tilde{w}_{2}:=\frac{1}{2}\left(\frac{1}{6}-\frac{(\gamma-1) N_{\mathrm{Pr}}}{8 \gamma}\right), \\
& \tilde{w}_{3}:=1+(\gamma-1) / N_{\mathrm{Pr}}^{1 / 2} .
\end{aligned}
$$

Note that $w_{1}, w_{2}, w_{3}, \tilde{w}_{1}, \tilde{w}_{2}$, and $\tilde{w}_{3}$ are all positive constants.

Approximating $g_{\beta}(z)$ and $g_{k}(z)$ by the first terms in the expansions (15) and (17) and substituting in Eqs. (10) and (11) leads to the following simple approximate formulas. For low-frequency and/or high flow resistivity $\left(\lambda_{A} \rightarrow 0\right),{ }^{10}$

$$
\beta_{b} \approx \frac{\gamma \Omega k}{k_{b}} \approx \frac{1}{2 s_{p}}\left(\frac{\gamma \Omega \omega \rho_{0}}{2 \sigma}\right)^{1 / 2}(1-i),
$$

while, for high frequency and/or for low flow resistivity $\left(\lambda_{A} \rightarrow \infty\right){ }^{9}$

$$
k_{b} \approx q, \quad \beta_{b} \approx \Omega / q .
$$

\section{B. Padé approximants}

The above formulas (15) and (17) provide good approximations for the admittance and wave-number functions $g_{\beta}$ and $g_{k}$ for small $z$ and large $z$. In this section we develop approximations accurate throughout the entire relevant range of $z$.

The asymptotic developments (15) and (17) suggest that we seek rational approximations for $g_{\beta}(z)$ and $g_{k}(z)$ in the form ${ }^{15}$

$$
g(z) \cong G(z)=\frac{\sum_{l=0}^{L} A_{l} z^{l}}{\sum_{m=0}^{M} B_{m} z^{m}}
$$

choosing the coefficients in these approximations to match the first few terms of the asymptotic expansions for $g_{\beta}(z)$ and $g_{k}(z)$ for small and large $z$, given in (15) and (17), and to give a good fit also for intermediate values of $z$ with $\arg z= \pm \pi / 4$. Specifically, if we adopt approximations $G_{\beta}(z)$ and $G_{k}(z)$ for $g_{\beta}(z)$ and $g_{k}(z)$, respectively, of the form (21) with $L=M=4$, we can choose the coefficients so that

$$
\begin{aligned}
& g_{\beta}(z)-G_{\beta}(z)=O\left(z^{5}\right), \\
& g_{k}(z)-G_{k}(z)=O\left(z^{3}\right), \quad \text { as } z \rightarrow 0,
\end{aligned}
$$

and

$$
\begin{aligned}
& g_{\beta}(z)-G_{\beta}(z)=O\left(z^{-2}\right), \\
& g_{k}(z)-G_{k}(z)=O\left(z^{-2}\right), \quad \text { as } z \rightarrow \infty,
\end{aligned}
$$

with $|\arg z|<\pi / 2$.

Note that the larger the values of $L$ and $M$ chosen, the more coefficients there are to adjust in (21), and the greater the accuracy that can be potentially achieved. (We must always have $L=M$, however, to obtain the correct asymptotic behaviour as $z \rightarrow \infty$.) It will be seen that with $L=M=4$ we obtain very accurate approximations $G_{\beta}$ and $G_{k}$, so that a larger choice for $L$ and $M$ seems unnecessary, while other calculations not reported here show that the choice $L=M=3$ leads to much reduced accuracy. Further, there is a theoretical advantage in adopting values $L=M \leqslant 4$. With this restricted range, and provided that the coefficients are real and non-negative in (21), with $A_{1}$ and $B_{1}$ in fact strictly positive (this will subsequently be seen to be true-see Table I), one can be sure that the approximation $G(z)$ has no zeros or poles in the sector of the complex plane $|\arg z| \leqslant \pi / 4$. This is a desirable property for the approximations $G_{\beta}(z)$ and $G_{k}(z)$ to have as it ensures that these functions and their inverses are analytic in the sector $|\arg z|<\pi / 4$, which in turn ensures that $\beta_{b}$ and $k_{b}$ and their inverses are analytic, considered as functions of $\omega$, in the half-plane $\operatorname{Im} \omega>0$. This is necessary to guarantee that causality is satisfied-see Rienstra ${ }^{16}$ and Miki. $^{2}$

The condition (22) ensures linear relationships between the coefficients in (21) and, in particular, implies that the approximations take the form 
TABLE I. Coefficient values appropriate to air for the rational approximants (24) and (25) to the admittance and wave-number functions.

\begin{tabular}{ccccc}
\hline \hline$m$ & $a_{m}$ & $b_{m}$ & $\tilde{a}_{m}$ & $\tilde{b}_{m}$ \\
\hline 1 & $\ldots$ & 0.487816 & 0.571125 & $\ldots$ \\
2 & 0.487816 & 0.272785 & 0.281620 & 0.571125 \\
3 & 0.176809 & 0.081597 & 0.080889 & 0.210930 \\
4 & 0.034778 & 0.014549 & 0.012106 & 0.040516 \\
\hline
\end{tabular}

$$
G_{\beta}(z)=w_{1} z \frac{1+a_{2} z+a_{3} z^{2}+a_{4} z^{3}}{1+b_{1} z+b_{2} z^{2}+b_{3} z^{3}+b_{4}}
$$

and

$$
G_{k}(z)=\frac{\tilde{w}_{1}}{z} \frac{1+\tilde{a}_{1} z+\tilde{a}_{2} z^{2}+\tilde{a}_{3} z^{3}+\tilde{a}_{4}}{1+\tilde{b}_{2} z+\tilde{b}_{3} z^{2}+\tilde{b}_{4} z^{3}}
$$

[ $w_{1}$ and $\tilde{w}_{1}$ defined by (16) and (18)].
Conditions (22) and (23) are insufficient to determine the coefficients in (24) and (25) uniquely and only ensure that the approximate admittance and wave-number functions $G_{\beta}(z)$ and $G_{k}(z)$ are highly accurate for small and large $z$. To ensure a good fit also for intermediate values of $z$, especially for $\arg z= \pm \pi / 4$, of importance when calculating $\beta_{b}$ and $k_{b}$ from expressions (10) and (11) with $e^{ \pm i \omega t}$ time dependence, we impose the additional conditions

$$
g_{\beta}\left(z_{0}\right)=G_{\beta}\left(z_{0}\right), \quad g_{\beta}\left(z_{0}^{*}\right)=G_{\beta}\left(z_{0}^{*}\right)
$$

and

$$
g_{k}\left(\tilde{z}_{0}\right)=G_{k}\left(\tilde{z}_{0}\right), \quad g_{k}\left(\tilde{z}_{0}^{*}\right)=G_{k}\left(\tilde{z}_{0}^{*}\right)
$$

for some points $z_{0}$ and $\tilde{z}_{0}$ with $\arg z_{0}=\arg \tilde{z}_{0}=-\pi / 4$.

The conditions (22), (23), (26), and (27) can be written as two sets of seven simultaneous linear equations for the unknown coefficients, namely

$$
\left[\begin{array}{ccccccc}
g_{0} & g_{0} z_{0} & g_{0} z_{0}^{2} & g_{0} z_{0}^{3} & -w_{1} z_{0} & -w_{1} z_{0}^{2} & -w_{1} z_{0}^{3} \\
g_{0}^{*} & g_{0}^{*} z_{0}^{*} & g_{0} z_{0}^{* 2} & g_{0}^{*} z_{0}^{* 3} & -w_{1} z_{0}^{*} & -w_{1} z_{0}^{* 2} & -w_{1} z_{0}^{* 3} \\
1 & 0 & 0 & 0 & -1 & 0 & 0 \\
0 & 1 & 0 & 0 & 0 & -1 & 0 \\
-w_{2} & 0 & 1 & 0 & 0 & 0 & -1 \\
0 & 0 & 0 & 1 & 0 & 0 & -w_{1} \\
0 & 0 & 1 & -w_{3} & 0 & -w_{1} & 0
\end{array}\right]\left[\begin{array}{c}
b_{1} \\
b_{2} \\
b_{3} \\
b_{4} \\
a_{1} \\
a_{2} \\
a_{3}
\end{array}\right]=\left[\begin{array}{c}
w_{1}-g_{0} / z_{0} \\
w_{1}-g_{0}^{*} / z_{0}^{*} \\
0 \\
w_{2} \\
0 \\
0 \\
0
\end{array}\right]
$$

and

$$
\left[\begin{array}{ccccccc}
\tilde{g}_{0} \tilde{z}_{0}^{2} & \tilde{g}_{0} \tilde{z}_{0}^{3} & \tilde{g}_{0} \tilde{z}_{0}^{4} & -\tilde{w}_{1} \tilde{z}_{0} & -\tilde{w}_{1} \tilde{z}_{0}^{2} & -\tilde{w}_{1} \tilde{z}_{0}^{3} & -\tilde{w}_{1} \tilde{z}_{0}^{4} \\
\tilde{g}_{0}^{*} \tilde{z}_{0}^{* 2} & \tilde{g}_{0}^{*} z_{0}^{* 3} & \tilde{g}_{0}^{*} \tilde{z}_{0}^{* 4} & -\tilde{w}_{1} \tilde{z}_{0}^{*} & -\tilde{w}_{1} \tilde{z}_{0}^{* 2} & -\tilde{w}_{1} \tilde{z}_{0}^{* 3} & -\tilde{w}_{1} \tilde{z}_{0}^{* 4} \\
1 & 0 & 0 & -1 & 0 & 0 & 0 \\
0 & 1 & 0 & 0 & -1 & 0 & 0 \\
\tilde{w}_{2} & 0 & 1 & 0 & 0 & -1 & 0 \\
0 & 0 & 1 & 0 & 0 & 0 & -\tilde{w}_{1} \\
0 & 1 & \tilde{w}_{3} & 0 & 0 & -\tilde{w}_{1} & 0
\end{array}\right]\left[\begin{array}{c}
\tilde{b}_{1} \\
\tilde{b}_{2} \\
\tilde{b}_{3} \\
\tilde{a}_{1} \\
\tilde{a}_{2} \\
\tilde{a}_{3} \\
\tilde{a}_{4}
\end{array}\right]=\left[\begin{array}{c}
\tilde{w}_{0}-\tilde{g}_{0} \tilde{z}_{0} \\
\tilde{w}_{0}^{*}-\tilde{g}_{0}^{*} \tilde{z}_{0}^{*} \\
0 \\
-\tilde{w}_{2} \\
0 \\
0 \\
0
\end{array}\right] .
$$

In these equations we have introduced the additional notations $g_{0}, g_{0}^{*}, \tilde{g}_{0}, \tilde{g}_{0}^{*}$, where

$$
\begin{array}{ll}
g_{0}:=g_{\beta}\left(z_{0}\right), & g_{0}^{*}:=g_{\beta}\left(z_{0}^{*}\right)=\left(g_{\beta}\left(z_{0}\right)\right)^{*}, \\
\tilde{g}_{0}:=g_{k}\left(\tilde{z}_{0}\right), & \tilde{g}_{0}^{*}:=g_{k}\left(\tilde{z}_{0}^{*}\right)=\left(g_{k}\left(\tilde{z}_{0}\right)\right)^{*} .
\end{array}
$$

All the elements of the coefficient matrices and righthand side vectors in (28) and (29) are real except for those in the first and second rows, and the elements in the second row, in view of (30), are just the complex conjugate of the elements in the first row. It is easy to see, therefore, that the solutions of (28) and (29) must be real, from which it follows that the approximate functions $G_{\beta}$ and $G_{k}$ satisfy the relations

$$
G_{\beta}\left(z^{*}\right)=\left(G_{\beta}(z)\right)^{*}, \quad G_{k}\left(z^{*}\right)=\left(G_{k}(z)\right)^{*},
$$

satisfied by the original functions $g_{\beta}$ and $g_{k}$.

For $\gamma=1.4$ and $N_{\mathrm{Pr}}=0.708$, values appropriate to air, the authors have solved the above systems for various choices of the points $z_{0}$ and $\tilde{z}_{0}$, with $\arg z_{0}=\arg \tilde{z}_{0}=-\pi / 4$, and have selected values $z_{0}=4.11 e^{-i \pi / 4}$ and $\tilde{z}_{0}=5.11 e^{-i \pi / 4}$ as ensuring good agreement between the approximate and exact admittance and wave-number functions. With these choices of $z_{0}$ and $\tilde{z}_{0}$ the solutions of (28) and (29) are shown in Table I.

Replacing $g_{\beta}$ and $g_{k}$ by the approximate admittance and wave-number functions $G_{\beta}$ and $G_{k}$ in (8)-(11), we obtain the following approximations to Attenborough's equations:

$$
\begin{aligned}
& \beta_{b}=(\Omega / q) G_{\beta}\left(\sqrt{-i} \lambda_{A}\right), \\
& k_{b} / k=q G_{k}\left(\sqrt{-i} \lambda_{A}\right),
\end{aligned}
$$

S. N. Chandler-Wilde and K. V. Horoshenkov: Porous media 


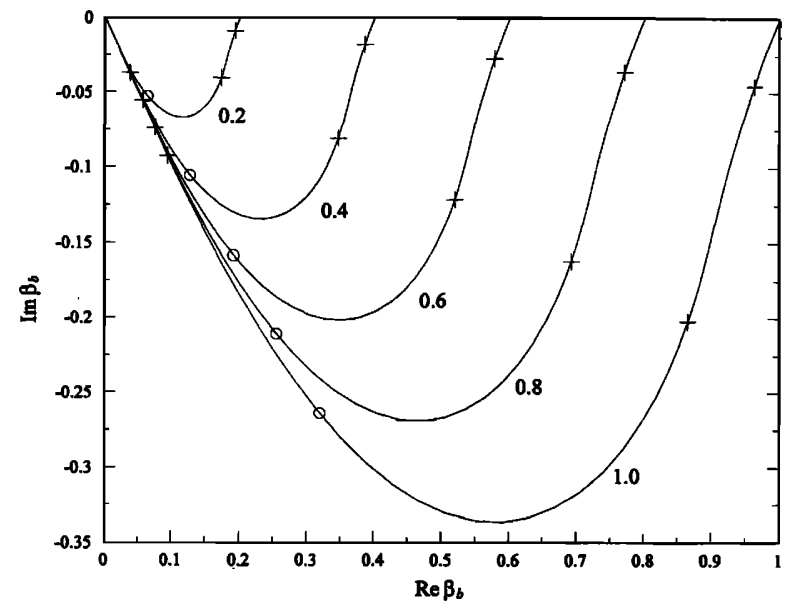

FIG. 1. Locus of $\beta_{b}=r g_{\beta}\left(\lambda_{A}\right)$ in the complex plane as $\lambda_{A}$ increases from 0 to $+\infty$. The parameter is the reduced porosity $r$. On each curve $\lambda_{A}$ increases by a factor of $\sqrt{10}$ on moving from one marker $(O$ or + ) to the next. $O$ marks the value of $\beta_{b}$ when $\lambda_{A}=1$.

$$
\begin{aligned}
& \rho_{b} / \rho_{0}=\left(q^{2} / \Omega\right) G_{\rho}\left(\sqrt{-i} \lambda_{A}\right), \\
& C_{b}=\left(\Omega / \gamma P_{0}\right) G_{C}\left(\sqrt{-i} \lambda_{A}\right) .
\end{aligned}
$$

In Eqs. (34) and (35) we have introduced approximate density and compressibility functions $G_{\rho}$ and $G_{C}$, also rational functions, defined by

$$
\begin{aligned}
& G_{\rho}(z):=G_{k}(z) / G_{\beta}(z), \\
& G_{C}(z):=G_{k}(z) G_{\beta}(z) .
\end{aligned}
$$

The other notations are as previously introduced, with $G_{\beta}$, $G_{k}$ defined by (24) and (25) and the coefficients as given in Table I.

As in the case of the original equations, (6), (7), (10), and (11), the above equations (32)-(35) are correct for $e^{-i \omega t}$ time dependence. Since $G_{\beta}$ and $G_{k}$ satisfy the relations (31), the formulas for $e^{i \omega t}$ time dependence are obtained simply by replacing $-i$ by $i$ in each equation.

The approximation (32) is particularly important for the case of sound propagation over a fluid-filled rigid frame porous half-space (for example, modeling outdoor sound propagation $\left.{ }^{10}\right)$. The normalized admittance of the surface of the porous half-space is simply $\beta_{b}$.

Figure 1 shows the locus of $\beta_{b}$ in the complex plane as $\lambda_{A}$ increases from 0 to $+\infty$, for various values of the parameter $r:=\Omega / q$, which will be referred to as the "reduced porosity." Since $0<\Omega<1$ and $q>1$, the reduced porosity satisfies $0<r<\Omega<1$. Thus, for all values of the physical parameters, the admittance $\beta_{b}$ lies in the region of the complex plane which lies between the real axis and the curve $g_{\beta}(\lambda): 0 \leqslant \lambda<\infty$. As can be seen from this diagram and (15), this region is contained in the sector " $-\pi / 4 \leqslant \arg \beta_{b} \leqslant 0$, $\left|\beta_{b}\right| \leqslant 1$.

Figures 2-5 show the errors in the approximate admittance and wave-number functions $G_{\beta}(z)$ and $G_{k}(z)$ on the line $\arg z=-\pi / 4$. [Since $G_{\beta}$ and $G_{k}$ satisfy the symmetry relations (31) as do the original functions $g_{\beta}$ and $g_{k}$ the errors have the same magnitude also on the line $\arg z=\pi / 4$.]
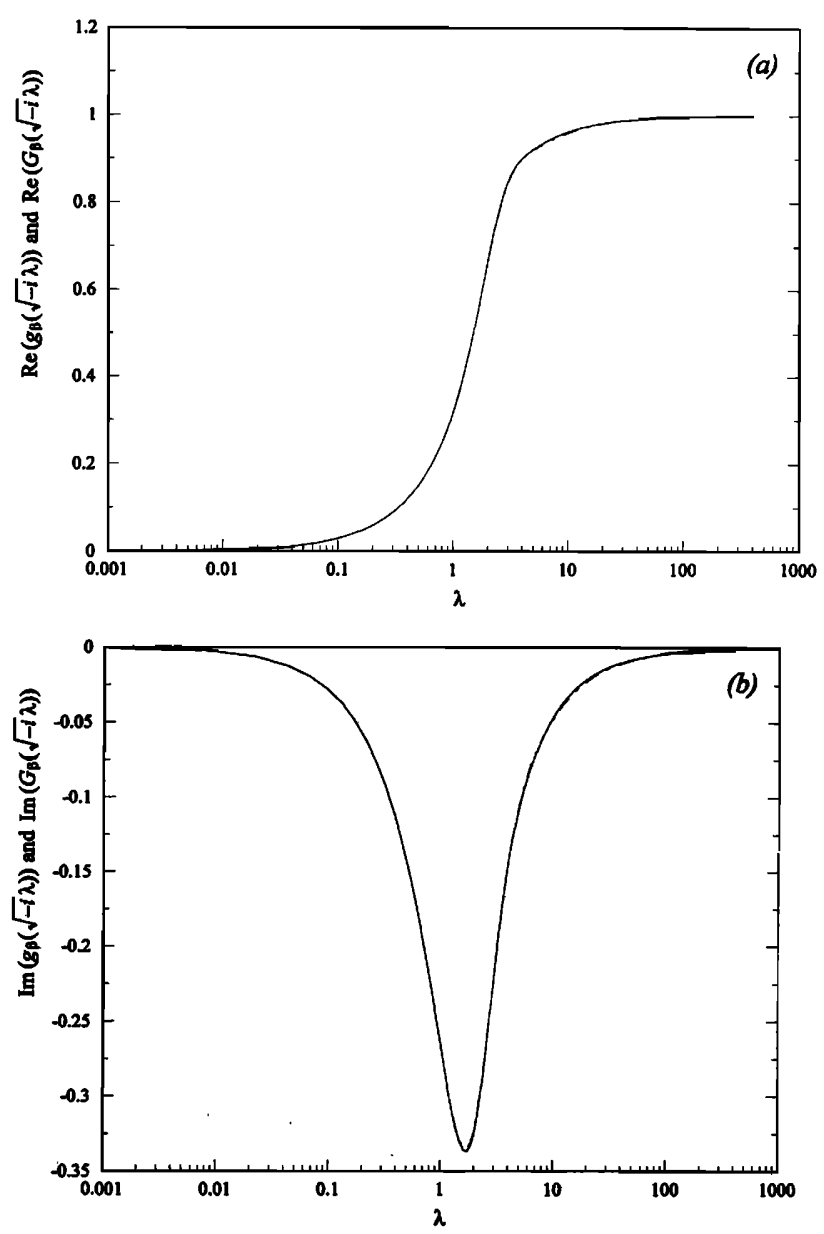

FIG. 2. Plot of the real and imaginary parts of $g_{\beta}(\sqrt{-i} \lambda)$ (—) and its approximation $G_{\beta}(\sqrt{-i} \lambda)(\cdot-\cdot)$ against $\lambda$. (a) Real parts; (b) imaginary parts.

The real and imaginary parts of $g_{\beta}(\sqrt{-i} \lambda)$ and $G_{\beta}(\sqrt{-i} \lambda)$ are compared in Fig. 2(a) and (b). In Fig. 3 $\Delta G_{\beta}(\sqrt{-i} \lambda)$ is plotted against $\lambda$, where $\Delta G_{\beta}(\sqrt{-i} \lambda)$ is the relative error in the admittance function, defined by

$$
\Delta G_{\beta}(z):=\left|\frac{g_{\beta}(z)-G_{\beta}(z)}{g_{\beta}(z)}\right| .
$$

In each of Figs. 2 and 3 it can be seen that, due to the conditions (22) and (23) which have been imposed, the approximation $G_{\beta}(\sqrt{-i} \lambda)$ is very accurate for small and large $\lambda$. Because of the condition (26), applied with $z_{0}$ $=4.11 \sqrt{-i}$, the approximation is also very accurate near $\lambda=4.11$. The largest errors are in the imaginary part of $G_{\beta}$ [see Fig. 2(b)], but the relative error does not exceed $3.4 \times 10^{-3}$ (i.e., $0.34 \%$ ) and is less than $2.0 \times 10^{-3}$ (i.e., $0.2 \%$ ) for $\lambda<1.5$ and $\lambda>20$ (see Fig. 3).

Figures 4 and 5 illustrate in a similar way the errors in the approximate wave-number function $G_{k}(\sqrt{-i} \lambda)$. Figure 4(a) and (b) compares the real and imaginary parts of $g_{k}(\sqrt{-i} \lambda)$ and $G_{k}(\sqrt{-i} \lambda)$, plotted against $\lambda$. In each of the graphs the curves corresponding to $g_{k}$ and to its approximation $G_{k}$ are indistinguishable. In Fig. $5 \Delta G_{k}(\sqrt{-i} \lambda)$ is plotted against $\lambda$, where $\Delta G_{k}(\sqrt{-i} \lambda)$ is the relative error in $G_{k}(z)$, defined by 


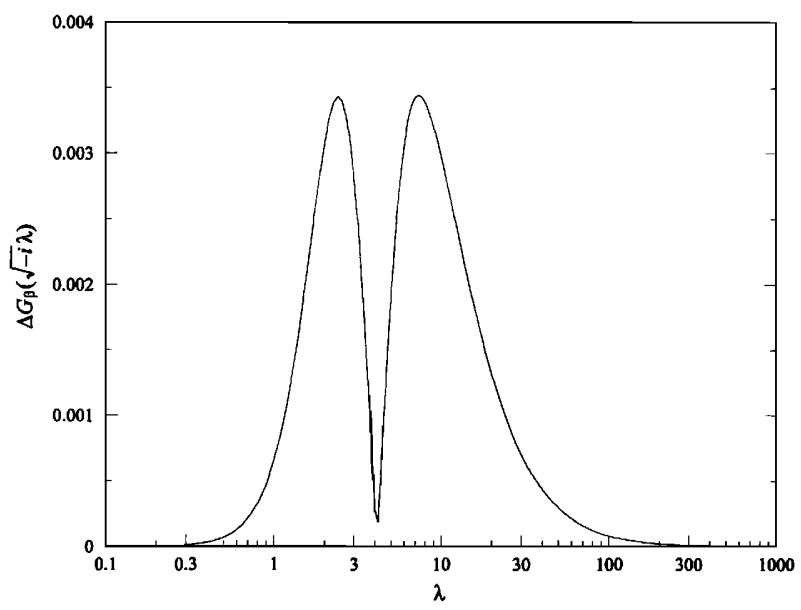

FIG. 3. Plot of relative error $\Delta G_{\beta}(\sqrt{-i} \lambda)$, in the approximation $G_{\beta}(\sqrt{-i} \lambda)$, against $\lambda$.

$$
\Delta G_{k}(z):=\left|\frac{g_{k}(z)-G_{k}(z)}{g_{k}(z)}\right| .
$$

Again, due to the conditions (22) and (23), the relative error is particularly small for small and large $\lambda$. Further, due to the condition (27) imposed with $\tilde{z}_{0}=5.12 \sqrt{-i}$, the error is also very small in the neighborhood of $\lambda=5.12$. For all
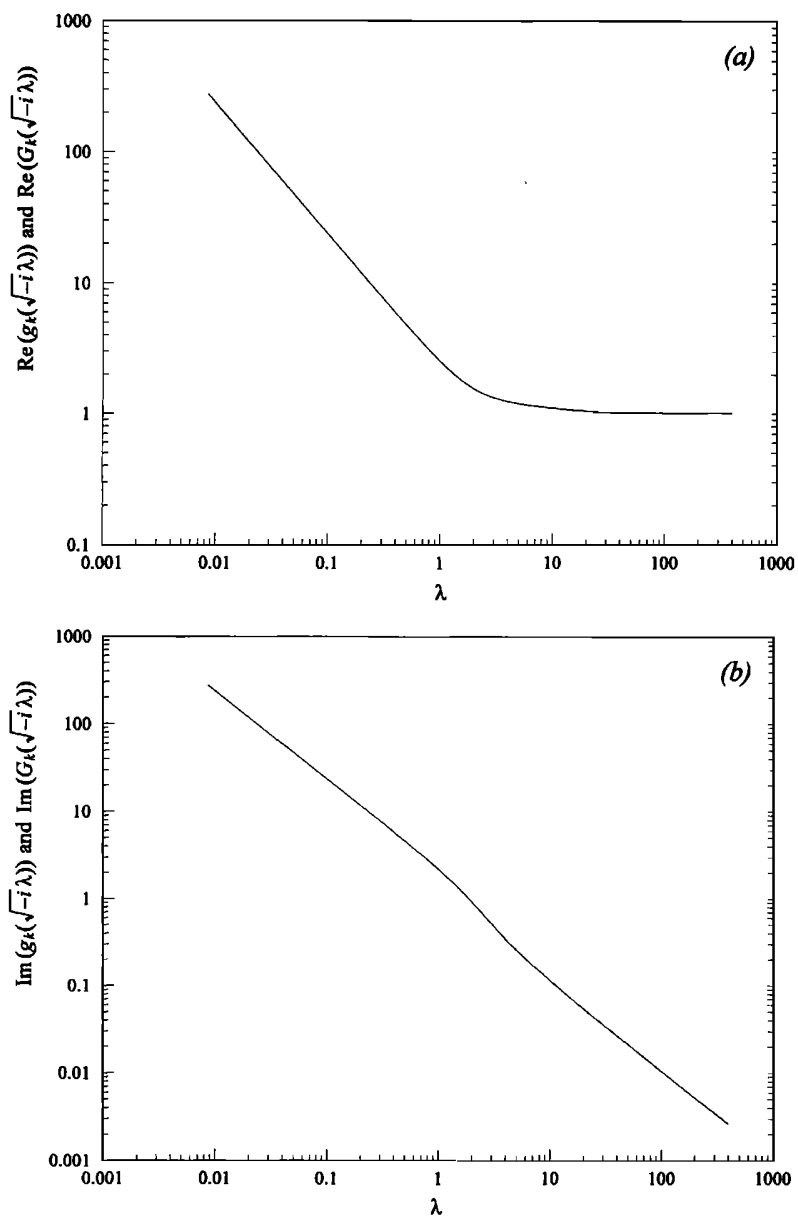

FIG. 4. Plots of the real and imaginary parts of $g_{k}(\sqrt{-i} \lambda)$ (—) and its approximation $G_{k}(\sqrt{-i} \lambda)(\cdot-\cdot-)$ against $\lambda$. (a) Real parts; (b) imaginary parts.

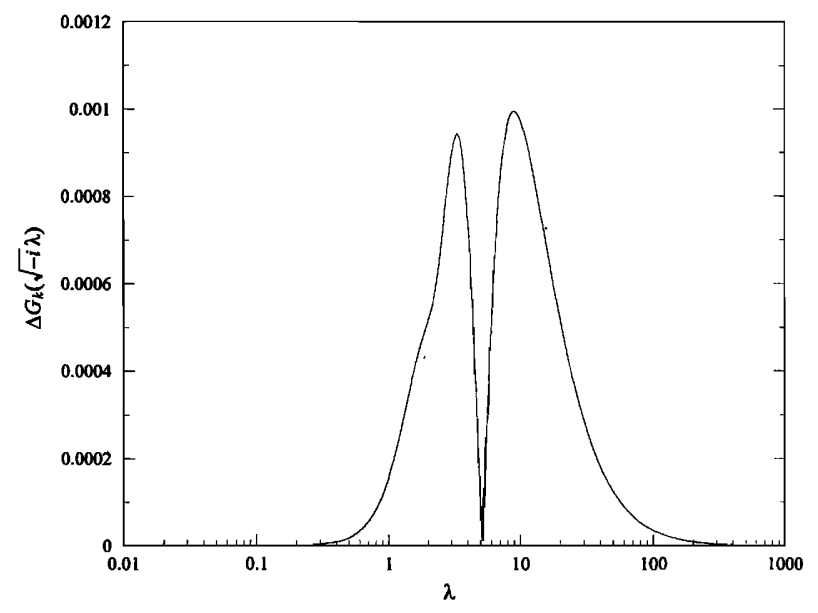

FIG. 5. Plot of relative error $\Delta G_{k}(\sqrt{-i} \lambda)$, in the approximation $G_{k}(\sqrt{-i} \lambda)$, against $\lambda$.

values of $\lambda$ the relative error is less than $10^{-3}(0.1 \%)$.

The computation of the admittance and wave-number functions $g_{\beta}$ and $g_{k}$, is particularly important in order to make, using the Attenborough model, predictions of acoustic wave propagation in porous media, and predictions of reflection and transmission coefficients for acoustic waves incident at a porous fluid-filled interface. It is less frequently important to compute the complex density $\rho_{b}$ and compressibility $C_{b}$ accurately. This being the case, the interpolation points $z_{0}$ and $\tilde{z}_{0}$ above were selected in order to minimize the errors in $G_{\beta}$ and $G_{k}$ rather than in the approximate density and compressibility functions $G_{\rho}$ and $G_{C}$.

These latter errors are shown in Figs. 6 and 7. In Figs. 6(a) and (b) the real and imaginary parts of the density function $g_{\rho}(\sqrt{-i} \lambda)$ and its approximation $G_{\rho}(\sqrt{-i} \lambda)$ are plotted against $\lambda$. It can be seen that the imaginary parts of $g_{\rho}(\sqrt{-i} \lambda)$ and $G_{\rho}(\sqrt{-i} \lambda)$ are almost indistinguishable: there is noticeable error in the real part of $G_{\rho}(\sqrt{-i} \lambda)$ for $0.4<\lambda<4.0$, but over most of this range the real part of $g_{\rho}(\sqrt{-i} \lambda)$ is in any case small compared to its imaginary part.

Figure 7(a) and (b) compares the real and imaginary parts of the compressibility function $g_{C}(\sqrt{-i} \lambda)$ and its approximation $G_{C}(\sqrt{-i} \lambda)$. Again the agreement, especially in the real part, is very good. There are slight differences in the imaginary parts around $\lambda=3$, but it can be noted that, for all $\lambda$, the imaginary part is a much smaller component.

\section{Impedance models for porous surfaces}

An important application of the Attenporough model is to provide a description of the frequency dependence of the acoustical impedance of porous fluid-filled surfaces. ${ }^{10}$ If the boundary is modeled as a homogeneous porous half-space then the normalized surface admittance is simply $\beta_{s}=\beta_{b}$, while if the boundary is modeled as a rigidly backed homogeneous porous layer of thickness $d$, the normalized surface admittance is ${ }^{10}$

$$
\beta_{s}=\beta_{b} \tanh \left(-i k_{b} d\right) .
$$



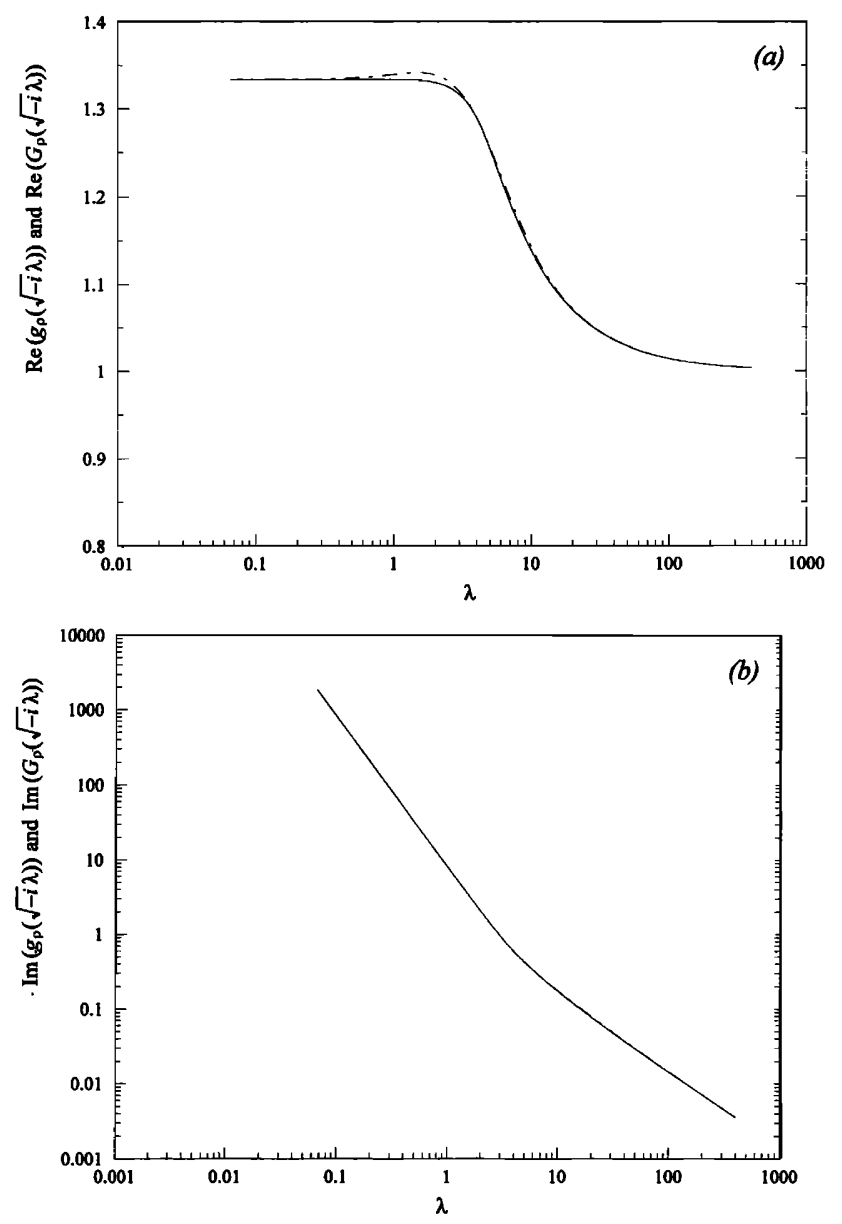

FIG. 6. Plots of the real and imaginary parts of $g_{\rho}(\sqrt{-i} \lambda)$ ( $\longrightarrow$ ) and its approximation $G_{\rho}(\sqrt{-i} \lambda)(\cdot-\cdot-)$ against $\lambda$. (a) Real parts; (b) imaginary parts.

More generally still, if the porous layer is backed by a porous half-space of normalized admittance (relative to air) $\beta_{b}^{\prime}$ and wave number $k_{b}^{\prime}$, the normalized surface admittance is

$$
\beta_{s}=\beta_{b}(1-\chi) /(1+\chi),
$$

with

$$
\chi=\exp \left(2 i k_{b} d\right)(1-\tau) /(1+\tau), \quad \tau=\beta_{b}^{\prime} / \beta_{b} .
$$

Clearly, this equation reduces to $\beta_{s}=\beta_{b}$ if $\beta_{b}^{\prime}=\beta_{b}$, or to Eq. (38) if $\beta_{b}^{\prime}=0$.

In many cases, for example, when modeling propagation over outdoor ground surfaces, it will be inconvenient to directly measure the parameters $\Omega, s_{p}, q$, and $\sigma$ in the Attenborough model, or impossible to obtain undisturbed samples, or samples which are representative of the area over which propagation is of interest. In these cases it is common to attempt to determine the parameters in the surface admittance model by acoustical measurements alone.

We have already remarked, at the beginning of Sec. I, following Sabatier et al. ${ }^{14}$ that acoustical measurement alone cannot determine all four parameters. For this reason, following Attenborough ${ }^{10}$ (and using the redefinition of the pore shape factor ratio in Attenborough ${ }^{11}$ ), we introduce an effective flow resistivity
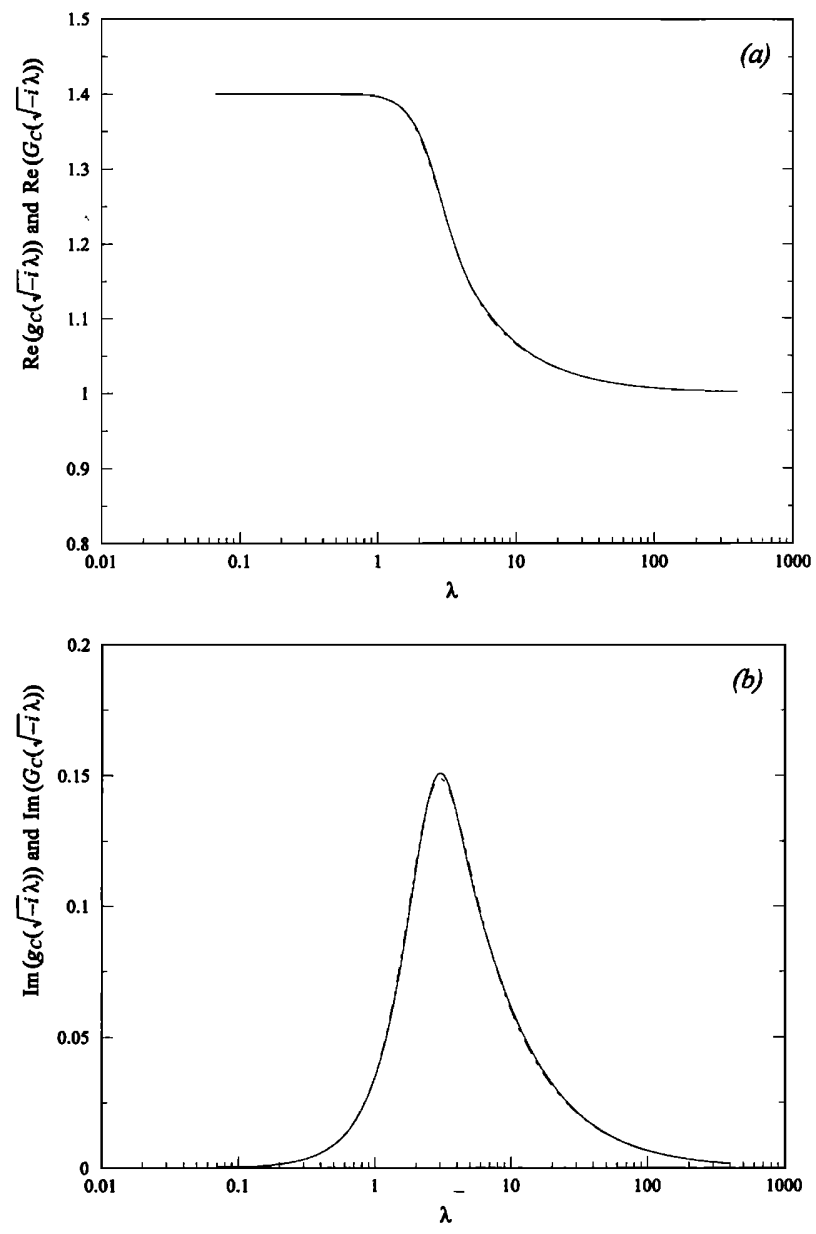

FIG. 7. Plots of the real and imaginary parts of $g_{C}(\sqrt{-i} \lambda)$ (-) and its approximation $G_{C}(\sqrt{-i} \lambda)(\cdot-\cdot-)$ againśt $\lambda$. (a) Real parts; (b) imaginary parts.

$$
\sigma_{e}=4 s_{p}^{2} \sigma / \Omega \text {. }
$$

In terms of this effective flow resistivity and the reduced porosity $r=\Omega / q$, and replacing the admittance function $g_{\beta}$ by its rational approximant $G_{\beta}$, from Eqs. (10) and (12) the surface admittance of a surface modeled as a porous halfspace is

$$
\beta_{s}=r G_{\beta}\left(\sqrt{-i} \lambda_{A}\right),
$$
with.

$$
\lambda_{A}=\left(8 \rho_{0} \omega / r^{2} \sigma_{e}\right)^{1 / 2}=(7.77 / r)\left(f / \sigma_{e}\right)^{1 / 2},
$$

where $f=\omega /(2 \pi)$, when the fluid is air of density $\rho_{0}=1.2 \mathrm{~kg}$ $\mathrm{m}^{-3}$. Clearly (40) and (41) form a two-parameter impedance model with parameters $r$ and $\sigma_{e}$.

From (19) we see that when $\lambda_{A}$ is small (40) reduces to

$$
\beta_{s}=\left(\gamma \rho_{0} \pi\right)^{1 / 2}\left(f / \sigma_{e}\right)^{1 / 2}(1-i)=2.30\left(f / \sigma_{e}\right)^{1 / 2}(1-i),
$$

this being the one-parameter ground impedance model proposed by Attenborough. ${ }^{10}$ From the first neglected term in (15a) (note that $w_{2}=0.0960$ for air) we estimate that this approximation is accurate to $\pm 10 \%$ provided $\lambda_{A}^{2} w_{2} \leqslant 0: 1$; that is, $f \leqslant 0.017 r^{2} \sigma_{e}$. For example, for outdoor sound propagation above a sandy soil (Ref. 10, Sec. 3.1), with 
$r \approx 0.2, \sigma_{e} \approx 300000 \mathrm{~N} \mathrm{~s} \mathrm{~m}^{-4}$, this criterion gives the limited range $f \leqslant 200 \mathrm{~Hz}$ as the range of validity of (42). In contrast, as we have seen in the previous section, (40) has high accuracy for all $\lambda_{A}$, but at the same time is very straightforward to compute. Equation (40) is also to be preferred to the two-parameter ground impedance model in Sabatier et al. ${ }^{14}$ based on approximations to the Attenborough model valid for small $\lambda_{A}$ as this model also has the wrong behavior at high frequency [compare Eq. (20) with the highfrequency limit given in Eq. (8) of Sabatier $e$ al.].

For large $\lambda_{A}$, from (20), Eq. (38) reduces to $\beta_{s}=r$. Thus we see that the high-frequency behavior of the surface impedance for a porous half-space is determined solely by the reduced porosity $r$, while the low-frequency behavior is determined only by $\sigma_{e}$.

Replacing the admittance and wave-number functions by their rational approximants $G_{\beta}$ and $G_{k}$, from Eqs. (32), (33), and (40), we see that the surface admittance of a surface modeled as a rigid-backed porous layer is

$$
\beta_{s}=r G_{\beta}\left(\sqrt{-i} \lambda_{A}\right) \tanh \left[-i k d_{e} G_{k}\left(\sqrt{-i} \lambda_{A}\right)\right],
$$

with $\lambda_{A}$ given by (41), and where we have introduced an effective depth $d_{e}=q d$. This is a three-parameter model, with parameters $r, \sigma_{e}$, and $d_{e}$, which is a very accurate approximation to the full Attenborough model for the surface impedance of a rigidly backed layer, throughout the frequency range and for all layer depths, in contrast, for example, to the two-parameter model given in Ref. 10, Eq. (33). At the same time, since $G_{\beta}$ and $G_{k}$ are given as the ratio of two polynomials, (43) is easy to compute.

Finally, if the ground is modeled as a porous layer of depth $d$ (effective depth $d_{e}=q d$ ), flow resistivity $\sigma_{e}$, and reduced porosity $r$, backed by a homogeneous half-space of effective flow resistivity $\sigma_{e}^{\prime}$ and reduced porosity $r^{\prime}$, the normalized surface admittance $\beta_{s}$ is given by

$$
\beta_{s}=r G_{\beta}\left(\sqrt{-i} \lambda_{A}\right)(1-\chi) /(1+\chi),
$$

with

$$
\begin{gathered}
\chi=\exp \left[2 i k d_{e} G_{k}\left(\sqrt{-i} \lambda_{A}\right)\right](1-\tau) /(1+\tau), \\
\tau=r^{\prime} G_{\beta}\left(\sqrt{-i} \lambda_{A}^{\prime}\right) /\left[r G_{\beta}\left(\sqrt{-i} \lambda_{A}\right)\right], \\
\lambda_{A}^{\prime}=\left(1 / r^{\prime}\right)\left(8 \rho_{0} \omega / \sigma_{e}^{\prime}\right)^{1 / 2},
\end{gathered}
$$

and $\lambda_{A}$ given by (41). This is a five-parameter model of ground impedance with parameters $r, \sigma_{e}, d_{e}, r^{\prime}$, and $\sigma_{e}^{\prime}$. It reduces to the two-parameter model (40) if $r=r^{\prime}$ and $\sigma_{e}$ $=\sigma_{e}^{\prime}$, and to the three-parameter model (43) if $r^{\prime}=0$.

\section{STINSON AND CHAMPOUX MODEL}

Based on the viscosity correction function $F$ given by Biot $^{17,18}$ and on a suggestion of Allard et al. ${ }^{19}$ Stinson and Champoux propose a modified version of the Attenborough model of the previous section which introduces a pore shape factor in a different and a more valid way.

From Stinson and Champoux [Ref. 4, Eqs. (33) and (64)], and changing their $e^{i \omega t}$ time dependence to a $e^{-i \omega t}$ time dependence for comparison with the previous section, the complex density of a bulk rigid frame porous material containing pores of uniform cross section is given by

$$
\rho_{b}=\left(q^{2} / \Omega\right) \rho_{0}-(\sigma / i \omega) F\left(\sqrt{-i} \lambda_{s}\right),
$$

where

$$
\lambda_{s}=s\left(8 \rho_{0} \omega q^{2} /(\Omega \sigma)\right)^{1 / 2},
$$

$s$ is the Stinson and Champoux shape factor, and the other variables are as previously defined (see List of Symbols). To unify the treatment of the $e^{-i \omega t}$ and $e^{i \omega t}$ time dependence cases we have modified the definition of the viscosity correction function. Our function $F$ is defined, for arbitrary complex $z$, by

$$
F(z):=\frac{1}{8} \frac{z^{2} S(z)}{1-S(z)},
$$

so that, from Eq. (1),

$$
F(z)=\frac{z I_{1}(z)}{4\left[I_{0}(z)-I_{1}(z)\right]} .
$$

It is related to the standard viscosity correction functions for $e^{i \omega t}$ and $e^{-i \omega t}$ time dependences, $F^{+}$and $F^{-}$, respectively, by

$$
F^{+}(\lambda)=F(\sqrt{i} \lambda), \quad F^{-}(\lambda)=F(\sqrt{-i} \lambda),
$$

for $\lambda>0$.

From Stinson and Champoux [Ref. 4, Eqs. (35) and (68)], the complex compressibility of the porous material is given by

$$
\begin{aligned}
C_{b}= & \left(\Omega / \gamma P_{0}\right)\left[\gamma-\rho_{0}(\gamma-1)\right. \\
& \left.\times\left[\rho_{0}-\sigma \Omega /\left(i \omega q^{2} N_{\mathrm{Pr}}\right) F\left(\sqrt{-i} \sqrt{N_{\mathrm{Pr}}} \lambda_{s}\right)\right]^{-1}\right] .
\end{aligned}
$$

Note that if we take the shape factor $s=1$ and the Attenborough shape factor $s_{p}=0.5$ (these values are recommended by Stinson and Champoux ${ }^{4}$ for circular pores), then Eqs. (45) and (49) simplify to give the same expressions for $\rho_{b}$ and $C_{b}$ as in the Attenborough model [Eqs. (6) and (7)]. For other combinations of shape factor values the two models give somewhat different predictions.

The only difficulty in computing with this model is calculating values of the viscosity correction function. We address this matter in the next section.

\section{A. Rational approximation of the complex viscosity function $F(z)$}

To approximate $F(z)$ let us consider its behavior for small and large $z$. From Eqs. (13) and (14) it follows that, as $z \rightarrow 0$,

$$
F(z)=1+\frac{1}{24} z^{2}+O\left(z^{4}\right),
$$

while, as $z \rightarrow \infty$,

$$
F(z)=z / 4+\frac{3}{8}+O\left(z^{-1}\right), \quad|\arg z|<\pi / 2 .
$$

Following the procedure of Sec. I, we approximate $F(z)$ by $F_{A}(z)$, where $F_{A}(z)$ has the form (21). It will be seen that the choice $L=3$ and $M=2$ provides a simple but accurate approximation if the coefficients are chosen to satisfy

$$
F(z)-F_{A}(z)=O\left(z^{4}\right), \quad \text { as } z \rightarrow 0,
$$




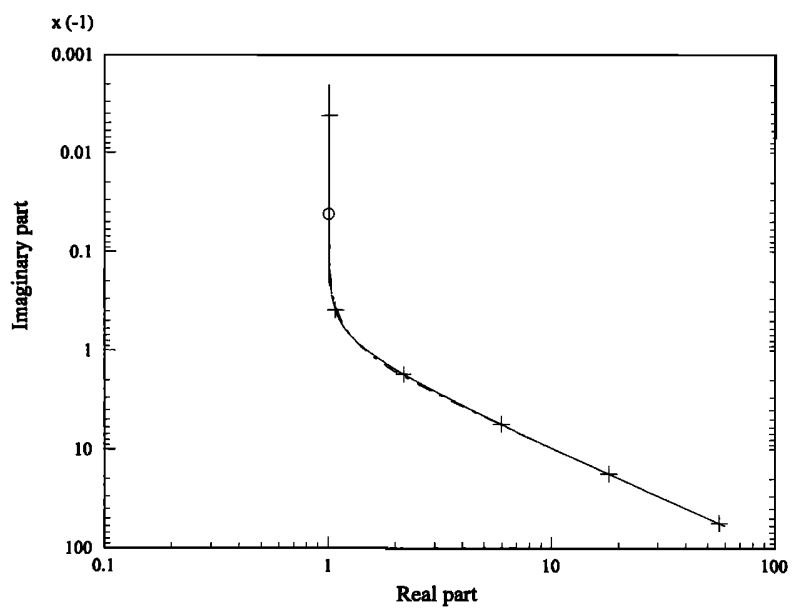

FIG. 8. Plot of $F(\sqrt{-i} \lambda)(-)$ and its approximation $F_{A}(\sqrt{-i} \lambda)$ (.-.-) in the complex plane as $\lambda$ increases from 0.26 to 400 . The markers + and $\bigcirc$ mark increases in $\lambda$ by a factor of $\sqrt{10}$, with $\bigcirc$ indicating $\lambda=1$.

and

$$
F(z)-F_{A}(z)=O\left(z^{-1}\right), \quad \text { as } z \rightarrow \infty,
$$

with $|\arg z|<\pi / 2$. Using the algorithm from the previous section we see that these conditions determine $F_{A}(z)$ to have the following simple form:

$$
F_{A}(z)=\frac{1}{8}\left(\frac{168+48 z+15 z^{2}+2 z^{3}}{21+6 z+z^{2}}\right) .
$$

Figure 8 shows the locus of $F(\sqrt{-i} \lambda)$ and its approximation $F_{A}(\sqrt{-i} \lambda)$ in the complex plane as $\lambda$ increases from 0.26 to 400 , and Fig. 9 plots the relative error $\Delta F_{A}(\sqrt{-i} \lambda)$ against $\lambda$, where $\Delta F_{A}$ is defined by

$$
\Delta F_{A}(z):=\left|\frac{F(z)-F_{A}(z)}{F(z)}\right| .
$$

It can be seen that, as expected, $F_{A}(\sqrt{-i} \lambda)$ is a very accurate approximation for small and large $\lambda$, accurate to less than $\pm 1 \%$ for $\lambda<2$ and $\lambda>30$. The error reaches a maximum value of less than $5 \%$ at $\lambda \approx 7$.

Obviously, the approximation $F_{A}$ can be used to generate approximations to the Stinson and Champoux model. Replacing $F$ by $F_{A}$ in (44) and (49), respectively, approximations for $\rho_{b}$ and $C_{b}$ are obtained. From these approximations the normalized characteristic admittance $\beta_{b}$ and wave number $k_{b}$ can be obtained, using Eqs. (8) and (9).

In Figs. 10-13 we compare the Stinson and Champoux model with these approximations, plotting against $\lambda_{s}$ the real and imaginary parts of $\beta_{b}$ and its approximation (Fig. 10), of $k_{b}$ and its approximation (Fig. 11), of $\rho_{b}$ (Fig. 12), and of $C_{b}$ (Fig. 13). In each graph the solid curve denotes the exact Stinson and Champoux model and the dash-dotted curve its approximation and we have chosen parameter values $q=1.26, \Omega=0.4, \gamma=1.4$, and $N_{\mathrm{Pr}}=0.708$ and shape factor $s=1$.

It can be noted that the graphs of the Stinson and Champoux formula and its approximation are almost coincident in the cases of the real and imaginary parts of the admittance and the wave number, the imaginary parts of the density, and

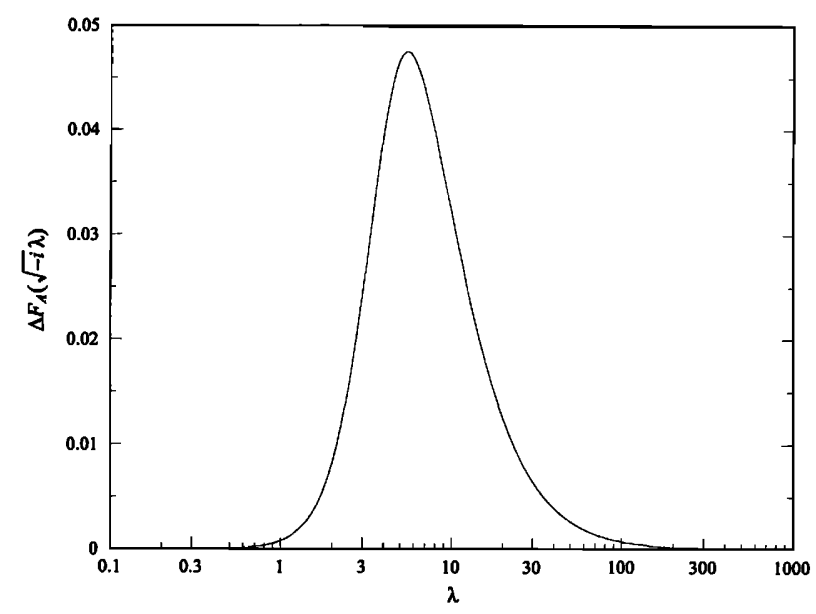

FIG. 9. Relative error $\Delta F_{A}\left(\sqrt{-i} \lambda\right.$ in the approximation $F_{A}(\sqrt{-i} \lambda)$ to the viscosity correction function $F(\sqrt{-i} \lambda)$ plotted against $\lambda$.

the real part of the compressibility. There are small differences between exact and approximate values in the range $1<\lambda_{s}<10$ in the cases of the real part of the density and the imaginary part of the compressibility. The real part of the density is much smaller than the imaginary part, and the imaginary part of the compressibility much smaller than the
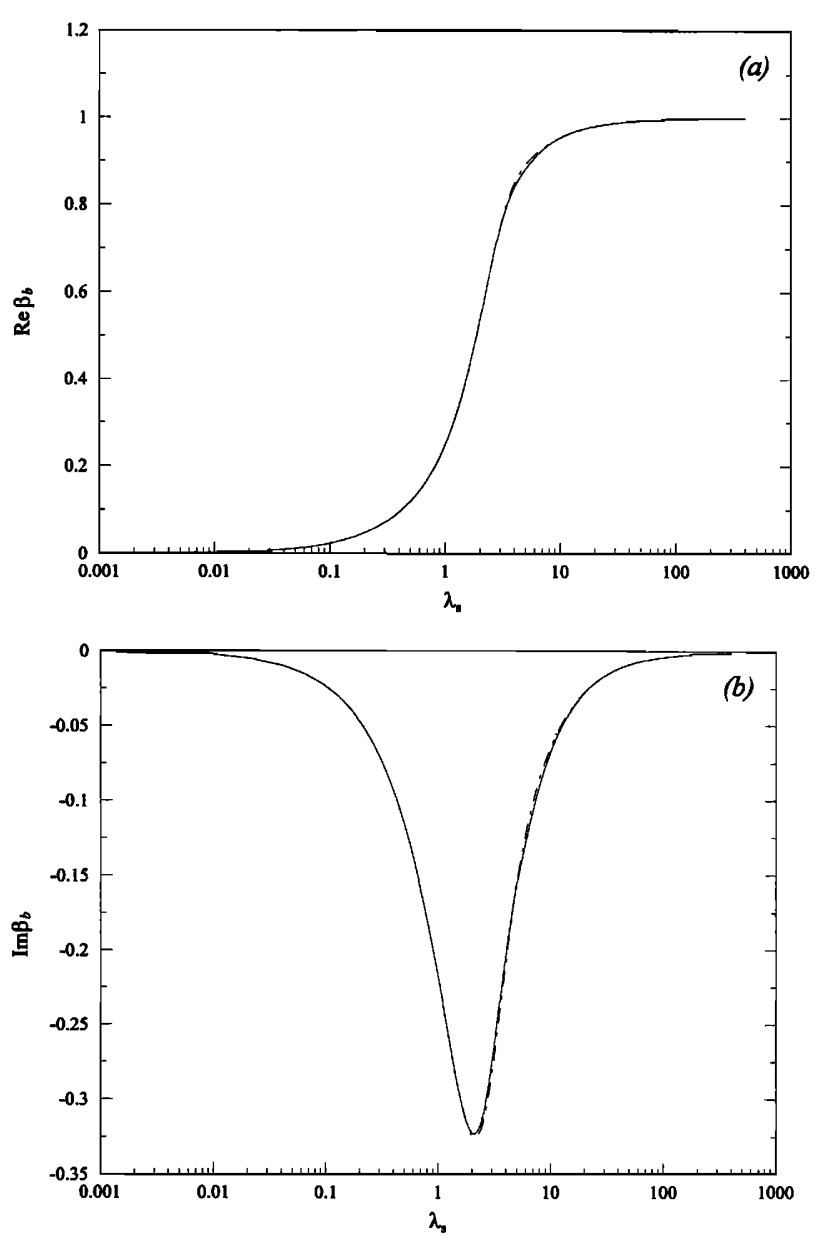

FIG. 10. Plots of the real and imaginary parts of the normalized admittance $\beta_{b}(\longrightarrow)$ and its approximation (.-.-) against $\lambda_{s}$. (a) Real parts; (b) imaginary parts. 

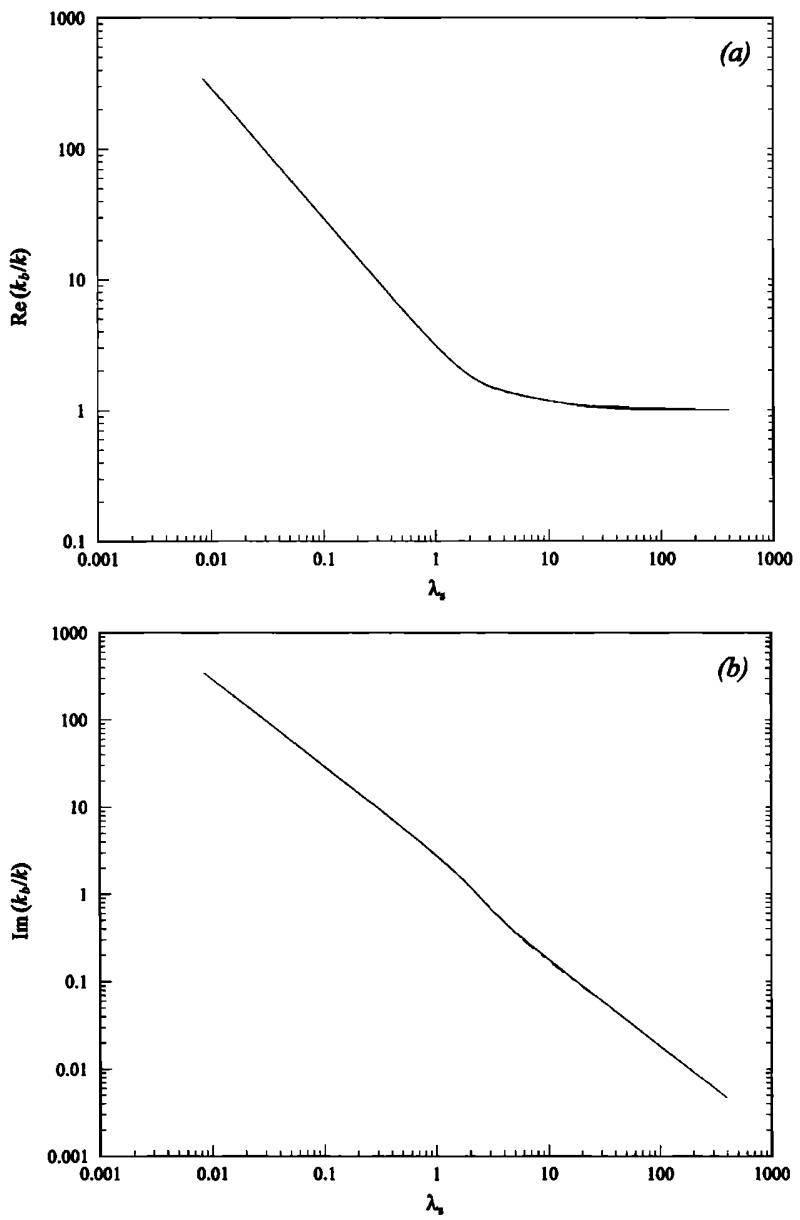

FIG. 11. Plots of the real and imaginary parts of the normalized wave number $k_{b} / k(\longrightarrow)$ and its approximation $(\cdot-\cdot-)$ against $\lambda_{s}$. (a) Real parts; (b) imaginary parts.

real part: it is therefore not surprising that a lower relative error is achieved in their computation.

\section{CONCLUSIONS}

Rational approximations for the functions occurring in two popular but somewhat complex models of the acoustical properties of rigid frame porous materials, namely those of Attenborough ${ }^{9-11}$ and Stinson and Champoux, ${ }^{4}$ have been presented in Eqs. (24), (25), (36), (37), and (54), respectively. These approximations simplify both models considerably and make computation with them at least an order of magnitude more efficient. This is particularly important when the models are used in an iterative computation, for example, in inverse problems of material parameter identification. The main equations of the paper are summarized for convenience of reference in the Appendix.

The approximations introduced are particularly accurate in the limits of high frequency/low flow resistivity and low frequency/high flow resistivity, but provide good accuracy for all parameter combinations, especially for the computation of complex characteristic admittance and complex wave number (see Figs. 4, 7, 11, and 12). In the opinion of the authors, the accuracy achieved is certainly sufficient for practical acoustical calculation, bearing in mind normal un-
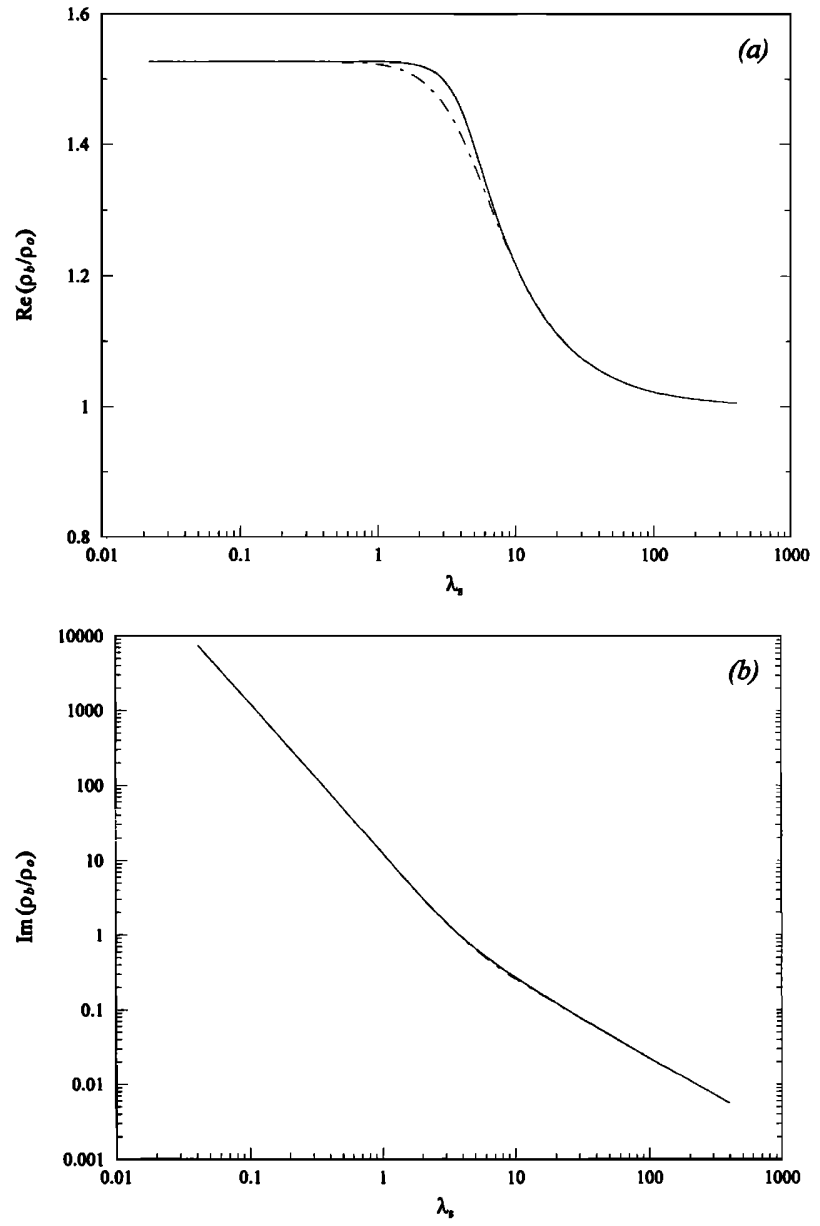

FIG. 12. Plots of the real and imaginary parts of the normalized density $\rho_{b} / \rho_{0}(\longrightarrow)$ and its approximation $(\cdot-\cdot-)$ against $\lambda_{s}$. (a) Real parts; (b) imaginary parts.

certainty in the material parameter values and, indeed, in the validity of the particular mathematical model of acoustical properties chosen.

In Sec. I C, based on the rational approximations to the Attenborough model presented, and on a model of a surface as a porous half-space, a simple but accurate model of surface impedance has been deduced, a two-parameter model with parameters $r=\Omega / q$ (the reduced porosity) and $\sigma_{e}$ (the effective flow resistivity introduced by Attenborough $\left.{ }^{10}\right)$. A three-parameter model, with extra parameter $d_{e}$, an effective depth, is also obtained, based on a model of the surface as a rigidly backed porous layer. Generalizing further, a fiveparameter equation [Eq. (44)] based on a model of the surface as a porous layer backed by a porous half-space is also deduced. (The five parameters are the reduced porosities and effective flow resistivities of the layer and half-space and the effective depth of the layer.) It is anticipated that these models will be of value in modeling the surface impedance of naturally occurring surfaces, for example, in outdoor sound propagation.

\section{APPENDIX: SUMMARY OF PROPOSED APPROXIMATIONS}

The following approximate formulas for the normalized characteristic admittance $\left(\boldsymbol{\beta}_{b}\right)$, dynamic complex wave num- 

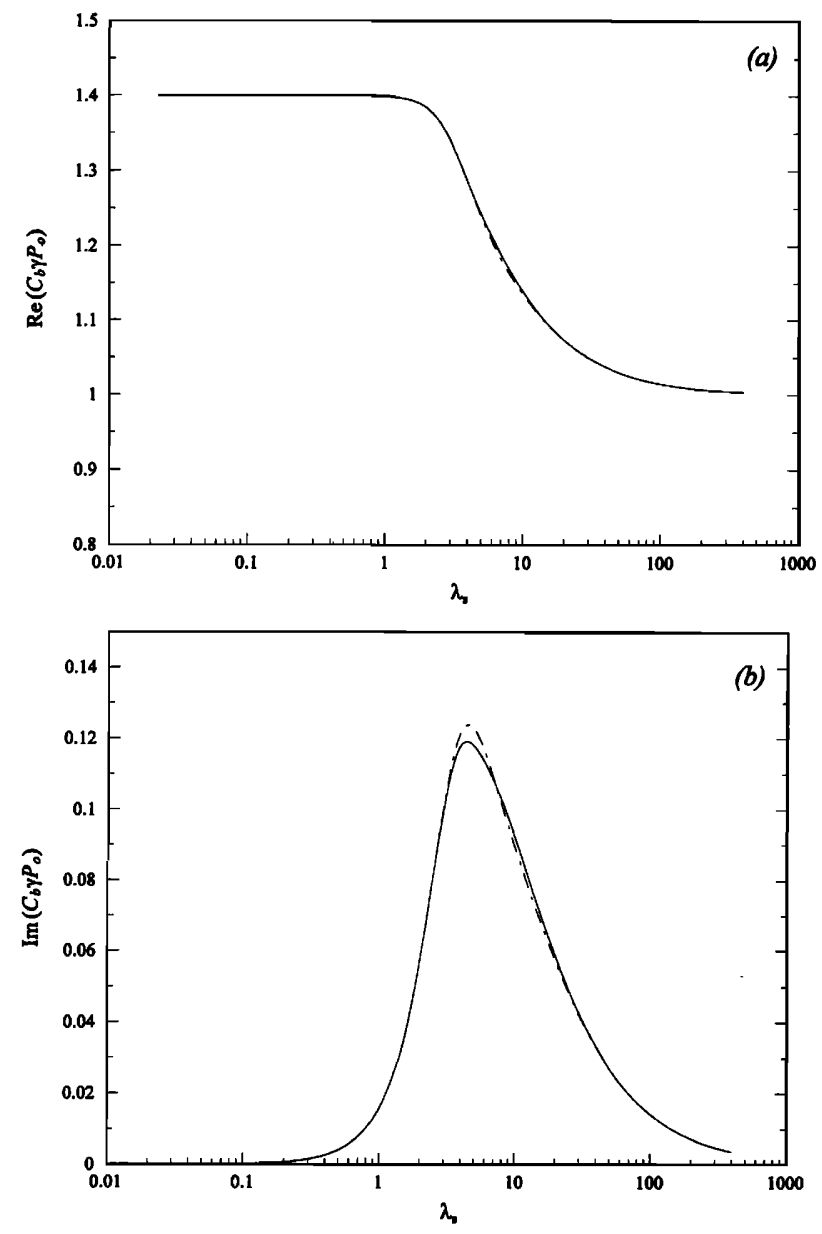

FIG. 13. Plots of the real and imaginary parts of the normalized compressibility $C_{b} \gamma P_{0}(\longrightarrow)$ and its approximation (.-.-) against $\lambda_{s}$. (a) real parts; (b) imaginary parts.

ber $\left(k_{b}\right)$, dynamic complex density $\left(\rho_{b}\right)$, and complex compressibility $\left(C_{b}\right)$ of rigid frame porous media have been derived in the paper. The formulas stated below are for $e^{-i \omega t}$ time dependence. To convert them to $e^{i \omega t}$ time dependence, replace $-i$ by $i$ in Eqs. (A1)-(A4), (A6), and (A7).

\section{Based on Attenborough model}

$$
\begin{aligned}
& \beta_{b}=(\Omega / q) G_{\beta}\left(\sqrt{-i} \lambda_{A}\right), \\
& k_{b} / k=q G_{k}\left(\sqrt{-i} \lambda_{A}\right), \\
& \rho_{b} / \rho=\left(q^{2} / \Omega\right) G_{\rho}\left(\sqrt{-i} \lambda_{A}\right), \\
& C_{b}=\left(\Omega / \gamma P_{0}\right) G_{C}\left(\sqrt{-i} \lambda_{A}\right),
\end{aligned}
$$

where

$$
\lambda_{A}=\left(1 / 2 s_{p}\right)\left(8 \rho_{0} q^{2} \omega / \Omega \sigma\right)^{1 / 2}
$$

and the functions $G_{\beta}$ and $G_{k}$ are defined by (24) and (25) with $w_{1}=\sqrt{\gamma / 8} \approx 0.41833, \tilde{w}_{1}=\sqrt{8 \gamma} \approx 3.34664$, and the other coefficient values as specified in Table I. The functions $G_{\rho}$ and $G_{C}$ are defined in terms of $G_{\beta}$ and $G_{k}$ by $G_{\rho}(z):=G_{k}(z) / G_{\beta}(z), G_{C}(z):=G_{k}(z) G_{\beta}(z)$.

\section{Based on Stinson and Champoux model}

$$
\begin{aligned}
\rho_{b}= & \left(q^{2} / \Omega\right) \rho_{0}-(\sigma / i \omega) F_{A}\left(\sqrt{-i} \lambda_{s}\right), \\
C_{b}= & \left(\Omega / \gamma P_{0}\right)\left[\gamma-\rho(\gamma-1)\left(\rho_{0}\right.\right. \\
& \left.\left.-\sigma \Omega /\left(i \omega q^{2} N_{\mathrm{Pr}_{\mathrm{r}}}\right) F_{A}\left(\sqrt{-i} \sqrt{N_{\mathrm{Pr}}} \lambda_{s}\right)\right)^{-1}\right], \\
k_{b}= & \omega\left(\rho_{b} C_{b}\right)^{1 / 2}, \quad \operatorname{Re} k_{b}>0, \\
\beta_{b}= & \left(\rho_{b} / \rho_{0} c C_{b}\right)^{1 / 2}, \quad \operatorname{Re} \beta_{b}>0,
\end{aligned}
$$

where

$$
\lambda_{s}=s\left(8 \rho_{0} \omega q^{2} /(\Omega \sigma)\right)^{1 / 2}
$$

and $F_{A}$, the approximation to the viscosity correction function, is defined by

$$
F_{A}(z)=\frac{1}{8}\left(\frac{168+48 z+15 z^{2}+2 z^{3}}{21+6 z+z^{2}}\right) .
$$

${ }^{1} \mathrm{~T}$. Yamamoto and A. Turgut, "Acoustic wave propagation through porous media with arbitrary pore size distributions," J. Acoust. Soc. Am. 83, 1744-1751 (1988)

${ }^{2}$ Y. Miki, "Acoustical properties of porous materials-Modifications of Delany-Bazley models," J. Acoust. Soc. Jpn. (E) 11, 19-28 (1990).

${ }^{3} \mathrm{M}$. R. Stinson, "The propagation of plane sound waves in narrow and wide circular tubes, and generalization to uniform tubes of arbitrary crosssectional shape," J. Acoust. Soc. Am. 89, 550-558 (1991).

${ }^{4} \mathrm{M}$. R. Stinson and Y. Champoux, "Propagation of sound and the assignment of shape factors of model porous materials having simple pore geometries," J. Acoust. Soc. Am. 91, 685-695 (1992).

5 J.-F. Allard and Y. Champoux, "New empirical equations for sound propagation in rigid frame fibrous materials," J. Acoust. Soc. Am. 91, 33463353 (1992).

${ }^{6} \mathrm{D}$. K. Wilson, "Relaxation-matched modelling of propagation through porous media, including fractal pore structure," J. Acoust. Soc. Am. 94, 1136-1145 (1993).

${ }^{7} \mathrm{~K}$. Attenborough, "Models for the acoustical properties of air-saturated granular media," Acta Acoust. 1, 213-226 (1993).

${ }^{8}$ M. E. Delany and E. N. Bazley, "Acoustical properties of fibrous absorbent materials," Appl. Acoust. 3, 105-116 (1970).

${ }^{9} \mathrm{~K}$. Attenborough, "Acoustical characteristics of rigid fibrous absorbents and granular materials," J. Acoust. Soc. Am. 73, 785-799 (1983).

${ }^{10} \mathrm{~K}$. Attenborough, "Acoustical impedance models for outdoor ground surfaces," J. Sound Vib. 99, 521-544 (1985).

${ }^{11} \mathrm{~K}$. Attenborough, "On the acoustic slow wave in air-filled granular media," J. Acoust. Soc. Am. 81, 93-102 (1987).

${ }^{12}$ C. Zwikker and C. W. Kosten, Sound Absorbing Materials (Elsevier, Amsterdam, 1949), Chap. 2.

${ }^{13}$ M. Abramovitz and I. A. Stegun, Handbook of Mathematical Functions (Dover, New York, 1969).

${ }^{14}$ J. M. Sabatier, R. Raspet, and C. K. Frederickson, "An improved procedure for the determination of ground parameters using level difference measurements," J. Acoust. Soc. Am. 94, 396-399 (1993).

${ }^{15}$ G. A. Backer, Jr., and P. R. Graves-Morris, Padé Approximants, Part II: Extensions and Applications (Cambridge U.P., Cambridge, 1984).

${ }^{16}$ S. W. Rienstra, "1-D reflection at an impedance wall," J. Sound Vib. 125, 43-51 (1988).

${ }^{17} \mathrm{M}$. A. Biot, "Theory of propagation of elastic waves in a fluid-saturated porous solid. I. Low-frequency range," J. Acoust. Soc. Am. 28, 168-178 (1956).

${ }^{18}$ M. A. Biot, "Theory of propagation of elastic waves in a fluid-saturated porous solid. II. Higher frequency range," J. Acoust. Soc. Am. 28, 179191 (1956).

${ }^{19}$ J. F. Allard, C. Depolier, J. Nicolas, W. Lauriks, and A. Cops, "Propriétés acoustiques des matériaux poreux saturés d'air et théorie de Biot," J. Acoust. 3, 29-38 (1990). 